\title{
$\beta$-elemene inhibits the generation of peritoneum effusion in pancreatic cancer via suppression of the HIF1A-VEGFA pathway based on network pharmacology
}

\author{
JUNQIU ZHU $^{1 *}$, BO LI $^{2 *}$, YONGSUO JI ${ }^{1}$, LINGLIN ZHU ${ }^{1}$, YANFEI ZHU ${ }^{1}$ and HONG ZHAO ${ }^{1}$
}

Departments of ${ }^{1}$ Oncology, ${ }^{2}$ Gastroenterology, Huadong Hospital Affiliated to Fudan University, Shanghai 200040, P.R. China

Received February 26, 2019; Accepted September 13, 2019

DOI: $10.3892 /$ or.2019.7360

\begin{abstract}
Pancreatic cancer remains one of the most lethal types of cancer. Late-stage pancreatic cancer patients usually suffer peritoneum effusion, which severely compromises quality of life. Great efforts have been made concerning the treatment of peritoneum effusion, including treatment with $\beta$-elemene. Although peritoneal perfusion of $\beta$-elemene attenuates the progression of malignant effusion without severe adverse effects in the clinic, the underlying molecular mechanism underlying the activity of $\beta$-elemene against peritoneum effusion remains unclear. In the present study, a network pharmacology approach was undertaken to explore the mechanism of $\beta$-elemene against peritoneum effusion. Particularly, the networks of $\beta$-elemene and pancreatic cancer target genes were constructed based on the BATMAN-TCM and DigSee databases, respectively. Thirty-three genes, including hypoxia inducible factor 1 subunit $\alpha$ (HIF1A), were discovered in both networks. A potential interaction of $\beta$-elemene with HIF1A was revealed by molecular docking simulation and co-expression analysis of pancreatic cancer datasets from The Cancer Genome Atlas (TCGA) database. Additionally, experimental validation by MTT assay demonstrated that $\beta$-elemene suppressed proliferation of PANC-1 and BxPC 3 cells and cells from peritoneum effusion in patients with pancreatic cancer. Furthermore, the protein expression levels of HIF1A and vascular endothelial growth factor A (VEGFA), as detected by western blotting, were reduced by $\beta$-elemene. Overall, this study proposes a potential molecular mechanism illustrating that $\beta$-elemene can block the HIF1A/VEGFA pathway, thereby inhibiting the generation of peritoneum effusion in pancreatic cancer based on network pharmacology analysis, and further
\end{abstract}

Correspondence to: Dr Hong Zhao, Department of Oncology, Huadong Hospital Affiliated to Fudan University, 221 West Yan'an Road, Jing'an, Shanghai 200040, P.R. China

E-mail: drzhao_hd@126.com

*Contributed equally

Key words: $\beta$-elemene, peritoneum effusion, HIF1A, VEGFA, pancreatic cancer, network pharmacology highlights the importance of targeting the HIF1A/VEGF pathway as a therapeutic approach to treat peritoneum effusion in patients with pancreatic cancer.

\section{Introduction}

Despite the tremendous improvements in cancer diagnosis and treatment, pancreatic cancer remains highly lethal worldwide (1). It is often diagnosed at advanced stages which is associated with a very poor patient prognosis (2). Surgical resection, chemotherapy and radiation therapy are the main treatments for pancreatic cancer. Nevertheless, most patients eventually experience recurrence, metastasis and resistance to chemotherapy and radiotherapy. Metastasis of pancreatic cancer often occurs in the liver, lung, bone and peritoneum (3). Peritoneal metastasis induces malignant peritoneum effusion, one of the most common symptoms of late-stage pancreatic cancer (4). In the clinic, chemotherapeutic drugs have been applied to attenuate peritoneum effusion with limited success (5). Therefore, a novel treatment option specifically targeting peritoneum effusion is urgently needed.

$\beta$-elemene (1-methyl-1-vinyl-2,4-diisopropenyl-cyclohexane), a compound isolated from an extract of the traditional Chinese medicinal herb Curcuma wenyujin, is a broad spectrum antitumor drug that has been approved by the state Food and Drug Administration of China for the treatment of specific solid and malignant tumors (6-8). Research has shown that $\beta$-elemene inhibits tumor cell proliferation (9), induces tumor cell apoptosis (10), suppresses tumor angiogenesis (11) and circumvents drug resistance $(12,13)$. Particularly, $\beta$-elemene emulsion plays significant roles in the clinical treatment of malignant effusion, such as pleural effusion in lung cancer (14). However, there are only a few reports of $\beta$-elemene use in the management of peritoneal effusion (15). Additionally, the mechanism of action behind this phenomenon remains unclear.

Intratumoral hypoxia is thought to be an essential characteristic of solid tumors, and results in the activation of hypoxia-inducible factor $1-\alpha$ (HIF1A). Previous studies have revealed that HIF1A plays critically important roles in maintaining the energy metabolism of tumor cells (16), tumor angiogenesis (17), accelerating tumor proliferation and metastasis (18). In addition, HIF1A can contribute to peritoneum effusion in several types of cancer via the promotion of its downstream target, vascular endothelial growth factor 
A (VEGFA) (19-21). Importantly, $\beta$-elemene can reduce the expression of HIF1A in cancer (22-24).

In the present study, network pharmacology was performed to investigate the molecular mechanism underlying the suppression of peritoneum effusion in pancreatic cancer by $\beta$-elemene. HIF1A was discovered as a target of both $\beta$-elemene and pancreatic cancer. $\beta$-elemene suppressed the proliferation of pancreatic cancer cells from peritoneum effusion. Furthermore, $\beta$-elemene attenuated the protein expression of HIF1A and its downstream target, VEGFA. These findings suggest that $\beta$-elemene ameliorates malignant peritoneum effusion in pancreatic cancer by inhibiting the HIF1A/VEGFA pathway.

\section{Materials and methods}

Tumor specimens and cell lines. Pancreatic cancer peritoneum effusion samples were obtained from two patients at Huadong Hospital Affiliated to Fudan University (Shanghai, China) for testing the cell viability. Fresh samples were collected from two patients upon obtaining written informed consent in Oct. 2018. The detailed information concerning these two patients are summarized in Table I. Cells were acquired from peritoneum effusion by centrifugation at $1,500 \mathrm{x} \mathrm{g}$ for $5 \mathrm{~min}$. This study was approved by the Internal Review and Ethics Boards of Huadong Hospital. The pancreatic cancer cell lines PANC-1 and BxPC3 were purchased from the American Type Culture Collection (ATCC; Manassas, VA, USA). Cells were cultured in RPMI-1640 medium, supplemented with $10 \%$ fetal bovine serum at $37^{\circ} \mathrm{C}$ with $5 \% \mathrm{CO}_{2}$.

Identification of $\beta$-elemene-associated genes. The human genes associated with $\beta$-elemene were acquired from the BATMAN-TCM (http://bionet.ncpsb.org/batman-tcm/) database. BATMAN-TCM is an online bioinformatics analysis tool specially designed for studying the molecular mechanisms of traditional Chinese medicine, and is based on traditional Chinese medicine ingredients' target prediction (25). The keyword '6918391', which is the PubChem CID (https://pubchem.ncbi.nlm.nih.gov/compound/6918391) of $\beta$-elemene, was input into the BATMAN-TCM database. To make the results more credible, the cutoff score was set at 5 .

Identification of pancreatic cancer-associated genes. The human genes associated with pancreatic cancer were acquired from the DigSee (http://210.107.182.61/geneSearch/) database. DigSee is a text mining search engine that provides evidence sentences describing which 'genes' are involved in the development of 'disease' through 'biological events' $(26,27)$. The keyword 'pancreatic neoplasms' was input into the DigSee database. To make the results more credible, the required number of academic papers was set to 5 .

Network construction. The Search Tool for the Retrieval of Interacting Genes (STRING, https://string-db.org/, version 10.5) database, which provides information regarding the predicted and experimental interactions of proteins, was used to obtain protein-protein interaction (PPI) data (28). Networks of $\beta$-elemene-target genes and pancreatic cancer-target genes were constructed using PPI analysis based on $\beta$-elemene-target genes and pancreatic cancer-target genes, respectively. The Kyoto Encyclopedia of Genes and Genomes (KEGG) pathway analysis of target genes in common between $\beta$-elemene and pancreatic cancer were performed using the Database for Annotation, Visualization and Integrated Discovery (DAVID, http://david.abcc.ncifcrf.gov/,version 6.8) database (29,30). A network of the common target genes of $\beta$-elemene and pancreatic cancer was constructed by linking PPI analysis and KEGG pathway analysis based on the common target genes. All networks were visualized utilizing Cytoscape software (31) (https://cytoscape.org/, version 3.4.0). In addition, the Cytoscape plugin Molecular Complex Detection (MCODE) was applied to analyze clustering modules in the network of target genes in common between $\beta$-elemene and pancreatic cancer.

Molecular docking simulation. The molecular docking simulation was conducted with AutoDockTools (32) (http://mgltools. scripps.edu/, version 1.5.6), which is an automated docking software designed to predict how small molecules bind to a receptor of known three dimensional (3D) structure, to find the preferred binding conformation of HIF1A and $\beta$-elemene. The human HIF1A structure was retrieved from the PDB database (PDB code: $1 \mathrm{H} 2 \mathrm{M}$ ). PubChem was referenced for the 3D structures of $\beta$-elemene. The preparation work included affixing hydrogen atoms and removing co-crystallized ligands as well as water molecules from the $1 \mathrm{H} 2 \mathrm{M}$. The detailed docking operation process strictly followed the software instruction manual. To cover all residues with grid map, each grid point in the $\mathrm{x}, \mathrm{y}$, and $\mathrm{z}$ axes was $126 \mathrm{x} 126 \mathrm{x} 126$ with $\mathrm{X}=21.796$, $\mathrm{Y}=28.466, \mathrm{Z}=30.538$ co-ordinates. In every docking test, 10 runs were executed with the population size set at 150 and a maximum number of evaluation of $2,500,000$. The remaining parameters were set to default. To visualize the 3D structure of the docking result, PyMOL software (33) (https://pymol.org/2/, version 2.2) was used.

Co-expression analysis and overall survival analysis. The Cancer Genome Atlas (TCGA) is a collaboration between the National Cancer Institute and the National Human Genome Research Institute that has generated comprehensive, multi-dimensional maps of the key genomic changes in 33 types of cancer (34). The dataset of pancreatic adenocarcinoma ( $N=177$, tumor samples with mRNA data) from TCGA was selected and a z-score threshold was set at \pm 2.0 . Genetic alteration and co-expression of HIF1A and VEGFA were analyzed using cBioPortal $(35,36)$ (http://www.cbioportal. org/). Analysis of overall survival (OS) was performed using Kaplan-Meier Plotter (37) (http://kmplot.com/analysis/).

Cell viability assay. PANC-1, BxPC3, and cells from two peritoneum effusion samples were seeded in 96-well plates at 5,000 cells/well in RPMI-1640 medium, supplemented with $10 \%$ fetal bovine serum at $37^{\circ} \mathrm{C}$ with $5 \% \mathrm{CO}_{2}$ and cultured overnight. Serial concentrations of $\beta$-elemene [CSPC Pharmaceutical Group Company Ltd. (YUANDA), Dalian, China] were added to the cells: $0,0.5,1,2,4,8$ and $16 \mu \mathrm{M}$. The stocking solution used here was prepared with an evenly-distributed aqueous solution of $\beta$-elemene, not the conventional emulsion dosage form of $\beta$-elemene. After $72 \mathrm{~h}$ 
Table I. Clinical characteristics of the pancreatic cancer patients.

\begin{tabular}{lccccccccc}
\hline Patient ID & $\begin{array}{c}\text { Age } \\
\text { (years) }\end{array}$ & Sex & Histology & Stage & $\begin{array}{c}\text { Tumor } \\
\text { size }(\mathrm{cm})\end{array}$ & $\begin{array}{c}\text { Vascular } \\
\text { invasion }\end{array}$ & Metastasis & $\begin{array}{c}\text { CA199 } \\
(\mathrm{kU} / \mathrm{l})\end{array}$ & $\begin{array}{c}\text { CEA } \\
(\mathrm{ng} / \mathrm{ml})\end{array}$ \\
\hline PC1 & 67 & Male & Adenocarcinoma & IV & $3.9 \times 3.7$ & Yes & Liver & 79.3 & 8.09 \\
PC2 & 78 & Male & Adenocarcinoma & IV & $3.2 \times 2.4$ & Yes & Liver & 289.08 & 5.85 \\
\hline
\end{tabular}

CA199, carbohydrate antigen 19-9; CEA, carcinoembryonic antigen.

of culture, the cells were incubated with $100 \mu \mathrm{l}$ of $0.5 \mathrm{mg} / \mathrm{ml}$ sterile MTT (3-(4,5-dimethyl-2-thiazolyl)-2,5-diphenyl-2H-tetrazolium bromide; Sigma-Aldrich; Merck KGaA) for $4 \mathrm{~h}$ at $37^{\circ} \mathrm{C}$. Then, the culture medium was removed and $150 \mu \mathrm{l}$ of DMSO (Sigma-Aldrich; Merck KGaA) was added. The absorbance values were measured at $490 \mathrm{~nm}$. The assay was performed in three replicates, and three parallel experiments were performed for each sample.

Western blotting. Total protein was extracted from PANC-1 and BxPC3 cells with radioimmunoprecipitation assay lysis buffer (Beijing Solarbio Science \& Technology Co., Ltd., Beijing, China) and quantified using a Bicinchoninic Acid Protein Assay Kit (Pierce; Thermo Fisher Scientific, Inc.). A total of $10 \mu \mathrm{g}$ protein was denatured and separated via sodium dodecyl sulfate-polyacrylamide gel electrophoresis (SDS-PAGE). The percentage of stacking gel and separation gel used in this study was 5 and $10 \%$, respectively. The proteins were transferred to polyvinylidene difluoride membranes (EMD Millipore), followed by blocking with $5 \%$ bovine serum albumin and incubation with primary antibodies (dilution 1:1,000) against GAPDH (cat. no. 2118; Cell Signaling Technology, Inc.), HIF1A (cat. no. 14179; Cell Signaling Technology, Inc.), and VEGFA (cat. no. ab52917; Abcam) overnight at $4^{\circ} \mathrm{C}$. The membranes were then incubated with secondary antibody (dilution 1:2,000) (cat. no. 7074; Cell Signaling Technology, Inc.) for $1 \mathrm{~h}$ at $37^{\circ} \mathrm{C}$. Finally, the membranes were imaged using the ChemiDOC ${ }^{\text {TM }}$ XRS system (Bio-Rad Laboratories, Inc.) following detection with an enhanced chemiluminescence kit (Beijing Solarbio Science \& Technology Co., Ltd.). The protein expression levels were calculated based on the greyscale values of the blots in Image (version 1.52; NIH) and normalized to that of GAPDH.

$R T-q P C R$ assay. Total RNA was extracted using the RNeasy mini kit (Qiagen) according to the manual without further modifications. RNA purity and quantity were assessed with NanoDrop 100 spectrophotometer (Thermo Scientific, Inc.). cDNA was synthesized with the Verso cDNA Synthesis kit (Thermo Scientific, Inc.) following the protocol described by the manufacturer. For qPCR, GoTap ${ }^{\circledR}$ qPCR Master Mix (Promega) was utilized to set up the reactions as suggested by the manufacturer. The sequences of the primers are: GAPDH (upstream GCACCGTCAAGGCTGAGAAC and downstream TGGTGAAGACGCCAGTGGA); HIF1A (upstream TCAAAGTCGGACAGCCTCAC and downstream ATC CATTGATTGCCCCAGCA); VEGFA (upstream TTGCAG

\section{ATGTGACAAGCCGA and downstream GGCCGCGGT GTGTCTA).}

Statistical analysis. SPSS statistical software (version 22.0; IBM Corp.) was used for statistical analysis. One-way analysis of variance followed by Tukey's multiple comparison post-hoc test was performed and a Student's t-test was used when only two groups were compared. Data are presented as the mean \pm standard deviation from three independent biological replicates. $\mathrm{P}<0.05$ was considered to be statistically significant.

\section{Results}

Network of $\beta$-elemene-target genes. To acquire a list of human genes associated with $\beta$-elemene, the BATMAN-TCM database was employed. The PubChem CID of $\beta$-elemene is 6918391. After entering the PubChem CID of $\beta$-elemene (6918391) as the keyword into the BATMAN-TCM database with a cutoff score $\geq 5,522 \beta$-elemene-target genes were obtained. A protein-protein interaction (PPI) network was built using the String database and Cytoscape software to uncover the relationships between these $\beta$-elemene-target genes. In total, 519 nodes and 3,672 edges were present in the network of $\beta$-elemene-target genes (Fig. 1).

Network of pancreatic cancer-target genes. The DigSee database was used to search for human genes relevant to pancreatic cancer. The required number of academic papers was set to be $>5$ on the website and 319 pancreatic cancer-target genes were obtained. Similarly, a PPI network was constructed to establish the relationship between these pancreatic cancer-target genes. A total of 318 nodes and 8,733 edges made up the network of the pancreatic cancer-target genes (Fig. 2).

Network of target genes for $\beta$-elemene against pancreatic cancer. To further uncover the potential pharmacological mechanisms of $\beta$-elemene activity against pancreatic cancer, target genes common to both $\beta$-elemene and pancreatic cancer were selected in both networks. A total of 33 genes belonging to both the $\beta$-elemene-target gene and pancreatic cancer-target gene networks were screened via Venn analysis (Fig. 3A). Additionally, KEGG pathway analysis based on the DAVID database was performed to determine the potential biological roles of these 33 genes. Subsequently, a network of target genes for $\beta$-elemene against pancreatic cancer was built using the String database and Cytoscape software (Fig. 3B). Two modules were identified by MCODE. Module 1 consisted 


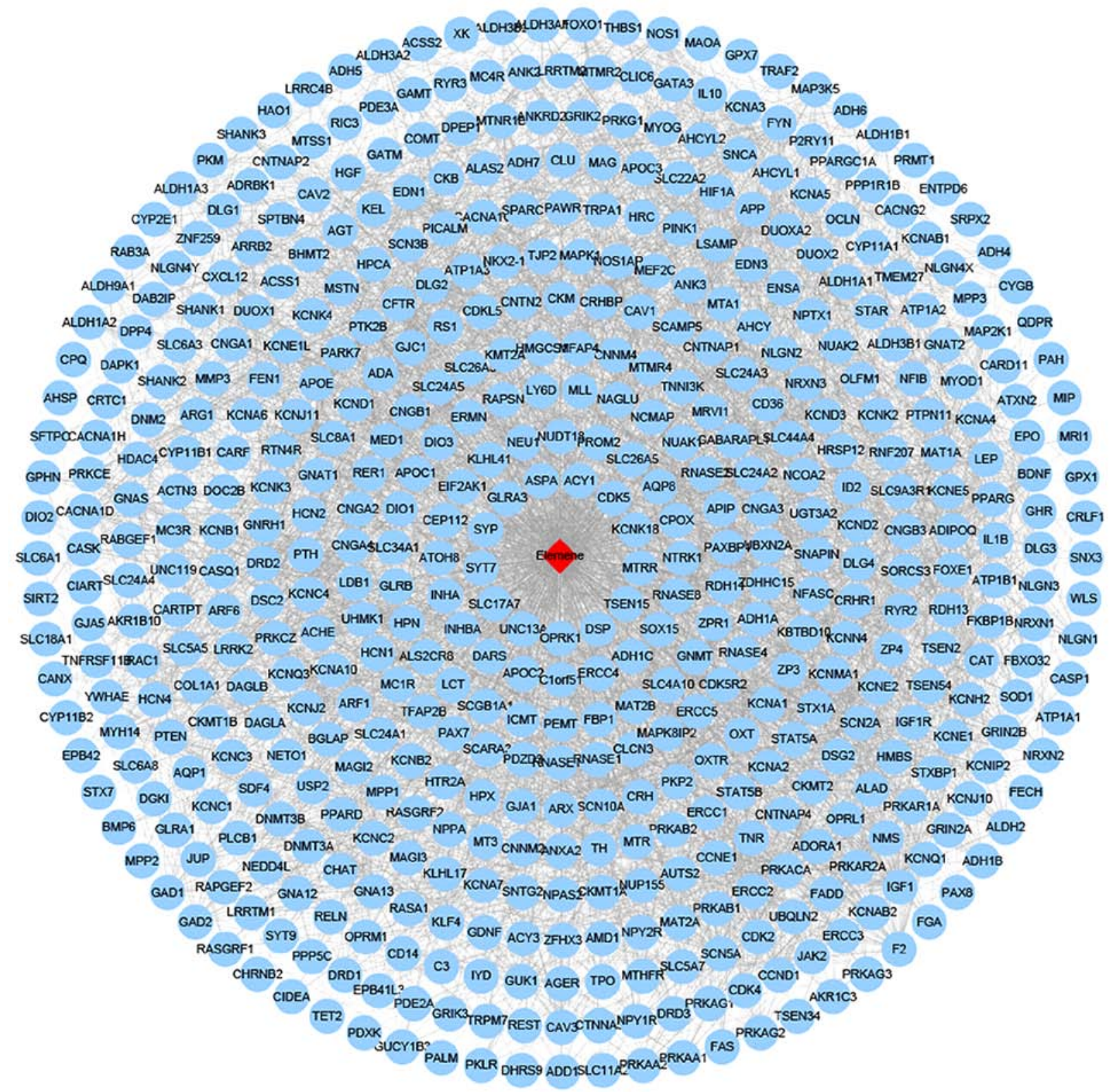

Figure 1. Network of $\beta$-elemene-target genes. Red diamond represents $\beta$-elemene; blue circles represent target genes of $\beta$-elemene.

of HIF1A, PTEN, PPARG, HGF, IL10, IL1B, JAK2, PTPN11, IGF1R, IGF1, MAPL1, MAP2K1 and CXCL12, and was highly relevant to proteoglycans in cancer, focal adhesions, and the HIF-1, PI3K-Akt, FoxO, Rap1, and Ras signaling pathways, among others. Module 2, containing CDK2 and CCND1, was of great importance in the PI3K-Akt, FoxO and p53 signaling pathways.

Molecular docking simulation of $\beta$-elemene to HIFlA. Molecular docking mimics the binding between molecules and their corresponding receptors. To provide deeper insight into the binding interactions between $\beta$-elemene and HIF1A, a molecular docking simulation was performed using AutoDockTools (http://mgltools.scripps.edu/, version 1.5.6) yielding a binding energy of $-5.3 \mathrm{kcal} / \mathrm{mol}$, a ligand efficiency of -0.35 and an inhibitor constant of $129.58 \mu \mathrm{M}$. Collectively, these results suggest the potential of direct binding between $\beta$-elemene and HIF1A (Fig. 4).
Co-expression of HIFIA and VEGFA. The expression profiles of HIF1A and VEGFA were not separate from each other. The dataset of pancreatic adenocarcinoma $(\mathrm{N}=177$, tumor samples with mRNA data) from TCGA was selected and analyzed with cBioPortal. HIF1A and VEGFA were both upregulated in pancreatic cancer (Fig. 5A). Furthermore, there was a trend of co-expression between HIF1A and VEGFA, verified by bioinformatic analysis. (Fig. 5B). Additionally, elevated expression of HIF1A and VEGFA corelated with poor overall survival, as evaluated using Kaplan-Meier Plotter (Fig. 5C and D).

Antitumor effect of $\beta$-elemene in pancreatic cancer. To investigate the antitumor effect of $\beta$-elemene in pancreatic cancer, an MTT assay was performed using PANC-1, BxPC3 cells and cells from the peritoneum effusion of two pancreatic cancer patients. The cells were treated with serial concentrations of $\beta$-elemene. Analysis of the PANC-1 and BxPC3 cells revealed that treatment with $\beta$-elemene reduced cell viability 


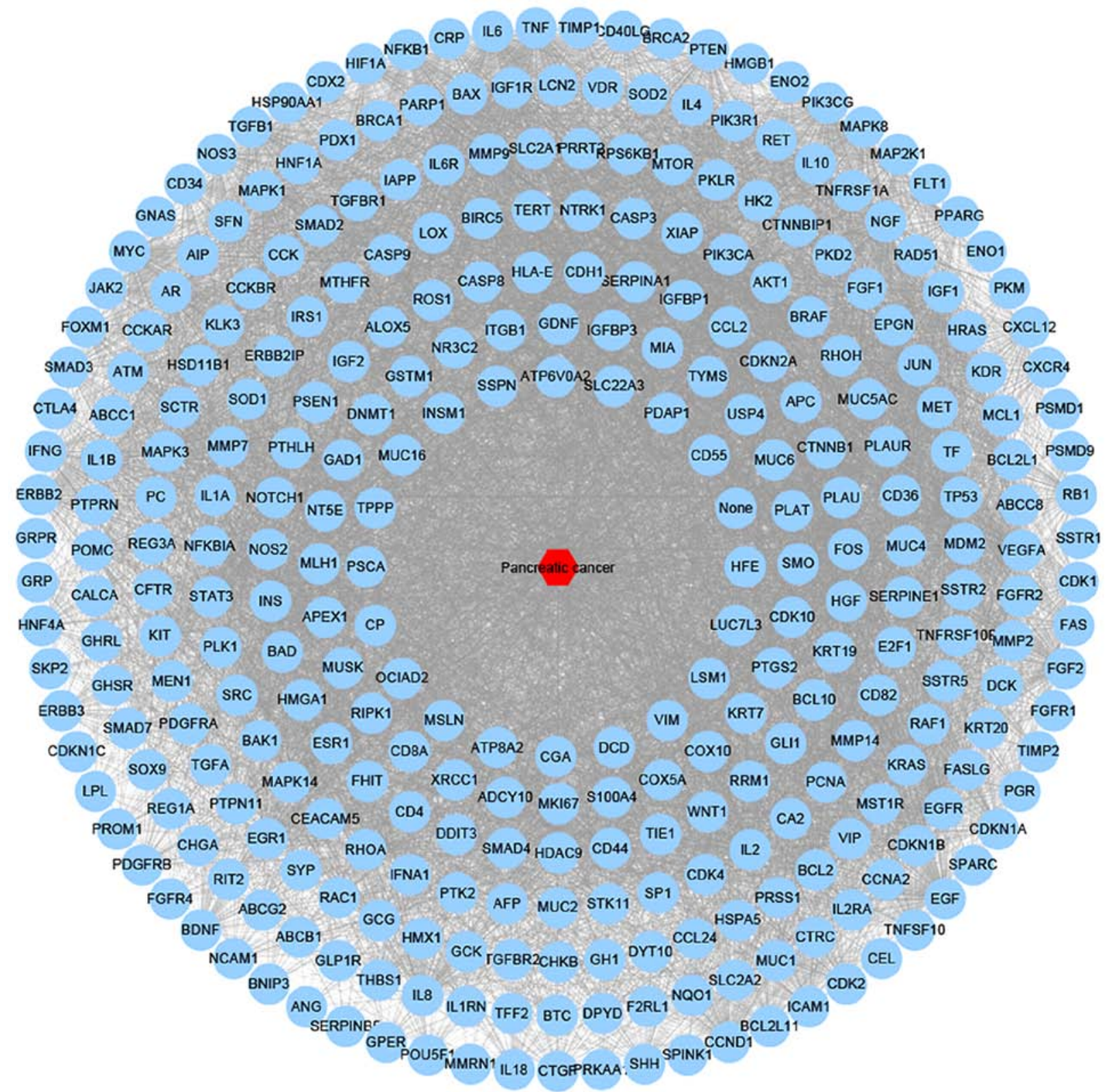

Figure 2. Network of pancreatic cancer-target genes. Red hexagon represents pancreatic cancer; blue circles represent target genes of pancreatic cancer.

(Fig. 6A and B). In addition, cells from the peritoneum effusion of two pancreatic cancer patients demonstrated decreased cell viability when treated with $\beta$-elemene (Fig. 6C and D). The $\mathrm{IC}_{50}$ values of $\beta$-elemene were $6.94 \pm 0.86,17.36 \pm 1.25$, $15.80 \pm 0.63,14.86 \pm 0.69 \mu \mathrm{M}$ in the PANC-1 and BxPC3 cell lines, and in the two peritoneum effusion samples, respectively. Collectively, $\beta$-elemene showed dose-dependent antitumor activity in pancreatic cancer.

Validation of HIF1A-VEGFA pathway inhibition by $\beta$-elemene in pancreatic cancer. Based on our network pharmacology analysis, $\beta$-elemene is expected to ameliorate malignant peritoneum effusion in pancreatic cancer by targeting the HIF1A/VEGFA pathway. Thus, an in vitro experiment was conducted to further explore the molecular mechanism of $\beta$-elemene against peritoneum effusion in pancreatic cancer. The HIF1A protein levels and those of its downstream target,
VEGFA, were evaluated via western blotting assay in both PANC-1 and BxPC3. Cells were treated with $\beta$-elemene at concentrations of $0,2,4$, and $8 \mu \mathrm{M}$. As the concentration of $\beta$-elemene increased, the expression of both HIF1A and VEGFA decreased, especially at the highest dosage (Fig. 7A and B), implying that $\beta$-elemene attenuates the expression of HIF1A, thereby inhibiting the expression of its downstream target, VEGFA. Apart from the evaluation of the protein expression, RT-qPCR was also conducted to assess expression of these genes at the RNA level. Similarly, the mRNA levels of HIF1A and VEGFA were decreased as the concentration of $\beta$-elemene increased in the PANC-1 and BxPC3 cell lines (Fig. 8), which was in concordance with the western blot assay. Taken together, all these results demonstrate that $\beta$-elemene functions to inhibit the HIF1A/VEGFA pathway, thereby conferring suppression of malignant peritoneum effusion in pancreatic cancer. 
A

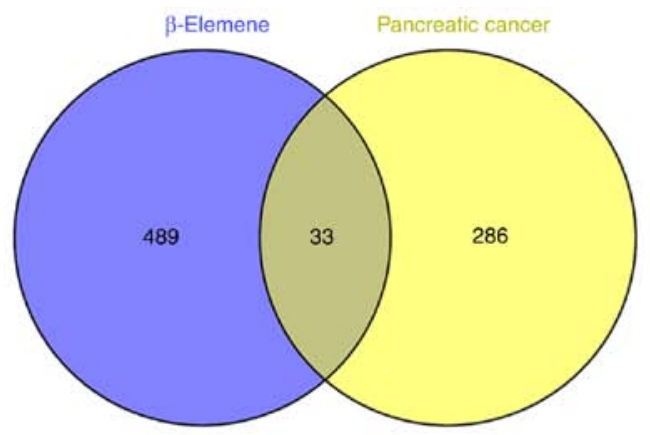

B

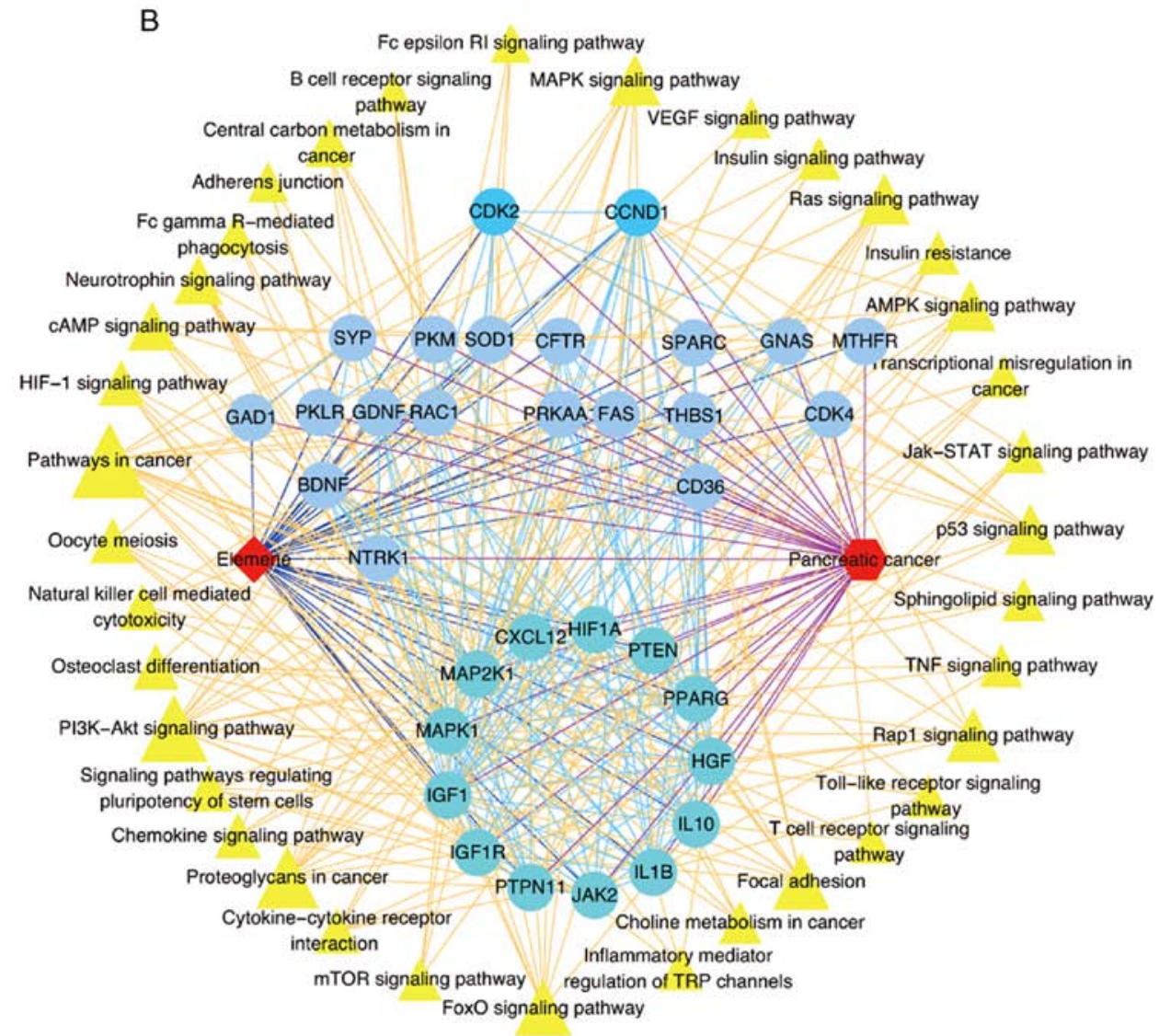

Figure 3. Thirty-three target genes for $\beta$-elemene against pancreatic cancer. (A) Venn diagram revealing the overlapping target genes for $\beta$-elemene against pancreatic cancer. (B) Network of target genes for $\beta$-elemene against pancreatic cancer. Red diamond represents $\beta$-elemene; red hexagon represents pancreatic cancer; blue circles represent target genes of both $\beta$-elemene and pancreatic cancer; yellow triangles represent KEGG pathway analysis of 33 target genes based on the DAVID database. KEGG, Kyoto encyclopedia of genes and genomes; DAVID, Database for Annotation, Visualization and Integrated Discovery.

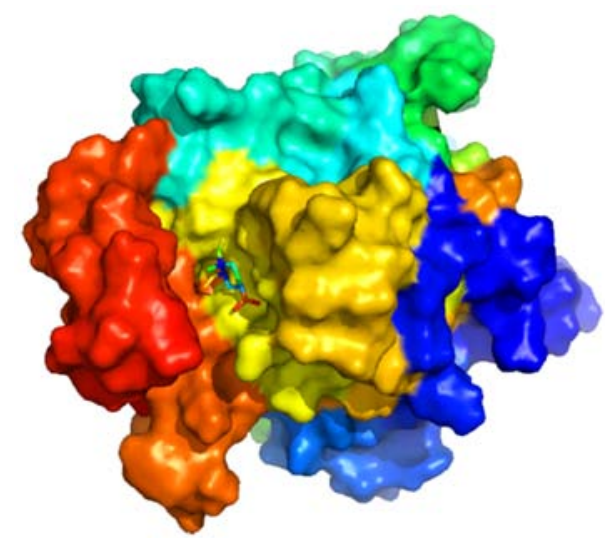

Figure 4. Molecular docking simulation of $\beta$-elemene to HIF1A. Sticks represent the three-dimensional structure of $\beta$-elemene; surface represents the protein structure of HIF1A. HIF1A, hypoxia-inducible factor 1- $\alpha$.

\section{Discussion}

$\beta$-elemene is a bioactive compound isolated from the traditional Chinese medicinal herb Curcuma wenyujin (38). It exerts a wide range of antitumor activities and has been approved in China to treat a wide spectrum of cancers including brain (9), breast (39), ovarian (40), gastric (41), hepatic (42) and lung cancers (43). A meta-analysis, including 46 clinical controlled trials with 2,992 patients, evaluated the efficacy and safety of $\beta$-elemene in treating malignant pleural effusion, and showed that $\beta$-elemene significantly improved the overall response rate in controlling malignant pleural effusion [risk ratio $(\mathrm{RR})=1.16$; $95 \%$ CI: 1.08-1.23; $\mathrm{P}<0.05)$ (14). Results of the study described herein revealed that $\beta$-elemene reduced the viability of PANC- 1 and BxPC 3 cell lines and cells from the peritoneum effusion of pancreatic cancer patients, rendering it a promising candidate for the management 


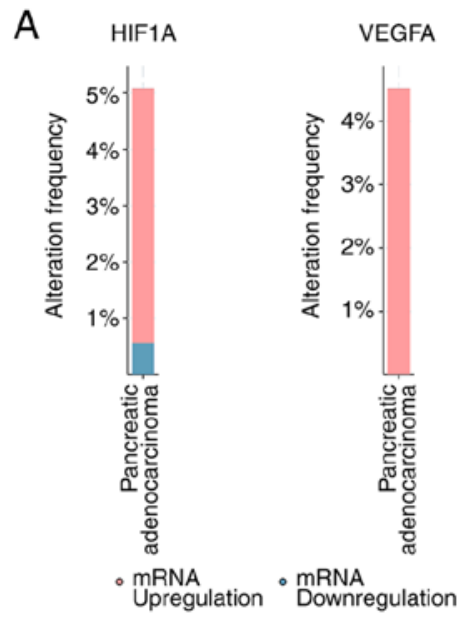

C

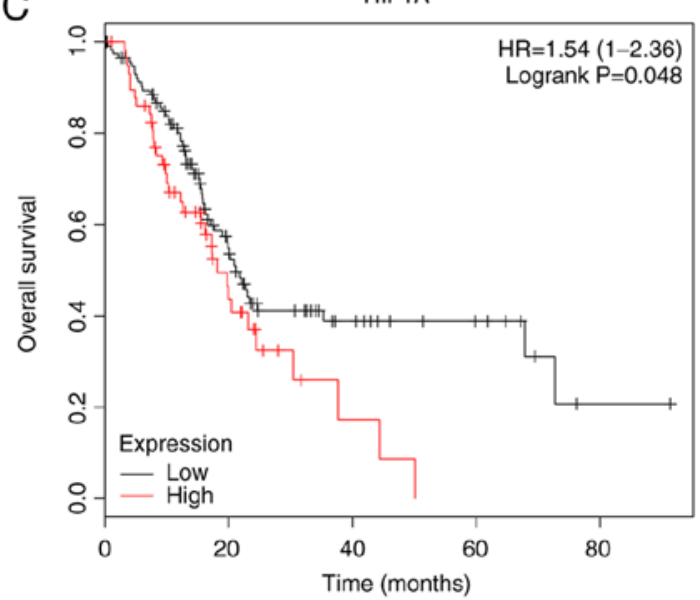

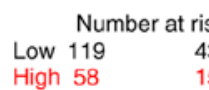

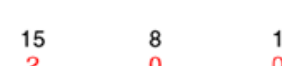

B

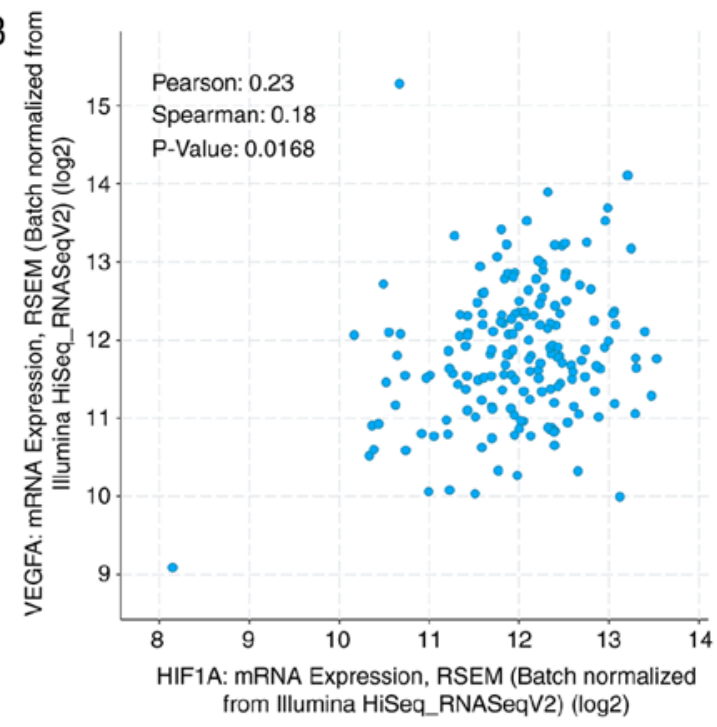

$\mathrm{D}$

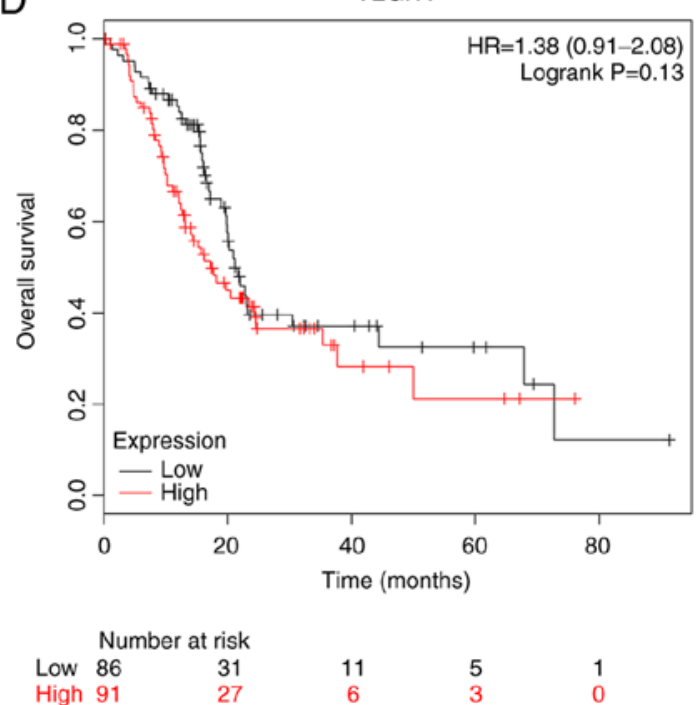

Figure 5. Analysis of HIF1A and VEGFA in the dataset of pancreatic adenocarcinoma (N=177, tumor samples with mRNA data) from TCGA. (A) Histogram of upregulation frequency of HIF1A and VEGFA in pancreatic cancer patients. (B) Scatter diagrams of co-expression relationship between HIF1A and VEGFA in pancreatic cancer patients. (C) Kaplan-Meier overall survival curves for all 177 pancreatic cancer patients stratified by high and low expression of HIF1A. (D) Kaplan-Meier overall survival curves for all 177 pancreatic cancer patients stratified by high and low expression of VEGFA. TCGA, The Cancer Genome Atlas; HIF1A, hypoxia-inducible factor 1- $\alpha$; VEGFA, vascular endothelial growth factor A.

of peritoneum effusion in pancreatic cancer patients. However, only preliminary in vitro studies were conducted to elucidate the potential of controlling the progression of peritoneum effusion in pancreatic cancer. More details should be addressed and the specific process by which $\beta$-elemene impacts the HIF1A/VEGFA pathway requires further exploration.

The advent of the big data era, the continuous accumulation of omics data, and the progress of bioinformatics methods provide strong support for network pharmacology development (44). As a core concept in network pharmacology, network targets have changed the current 'single target' research approach and provided a potential research strategy for analyzing the biological basis of traditional Chinese medicine from the perspective of networks and guiding the discovery of new active ingredients in traditional Chinese medicine $(45,46)$. Several studies have applied the network pharmacology approach to predict the potential targets of traditional Chinese medicine compounds.
HIF1A was discovered as one of the 31 target proteins that might be regulated by active ingredients in the Fuzheng Huayu formula in inhibiting hepatic stellate cell viability based on network pharmacology analysis (47). In addition, a network pharmacology approach determined that HIF1A is one of the most important potential protein targets of tetramethylpyrazine hydrochloride and paeoniflorin in the Chuanxiong-Chishao herb-pair for promoting angiogenic activity (48). In our study, HIF1A was identified as a target of $\beta$-elemene using the BATMAN-TCM database, as well as a target of pancreatic cancer using the DigSee database. Particularly, experimental validation by MTT assay and western blot analysis showed that the protein expression of HIF1A was hindered along with decreased cell viability following $\beta$-elemene treatment in pancreatic cancer cells. Taken together, network pharmacology analysis provided a theoretical direction for the exploration of a molecular mechanism of $\beta$-elemene against peritoneum 

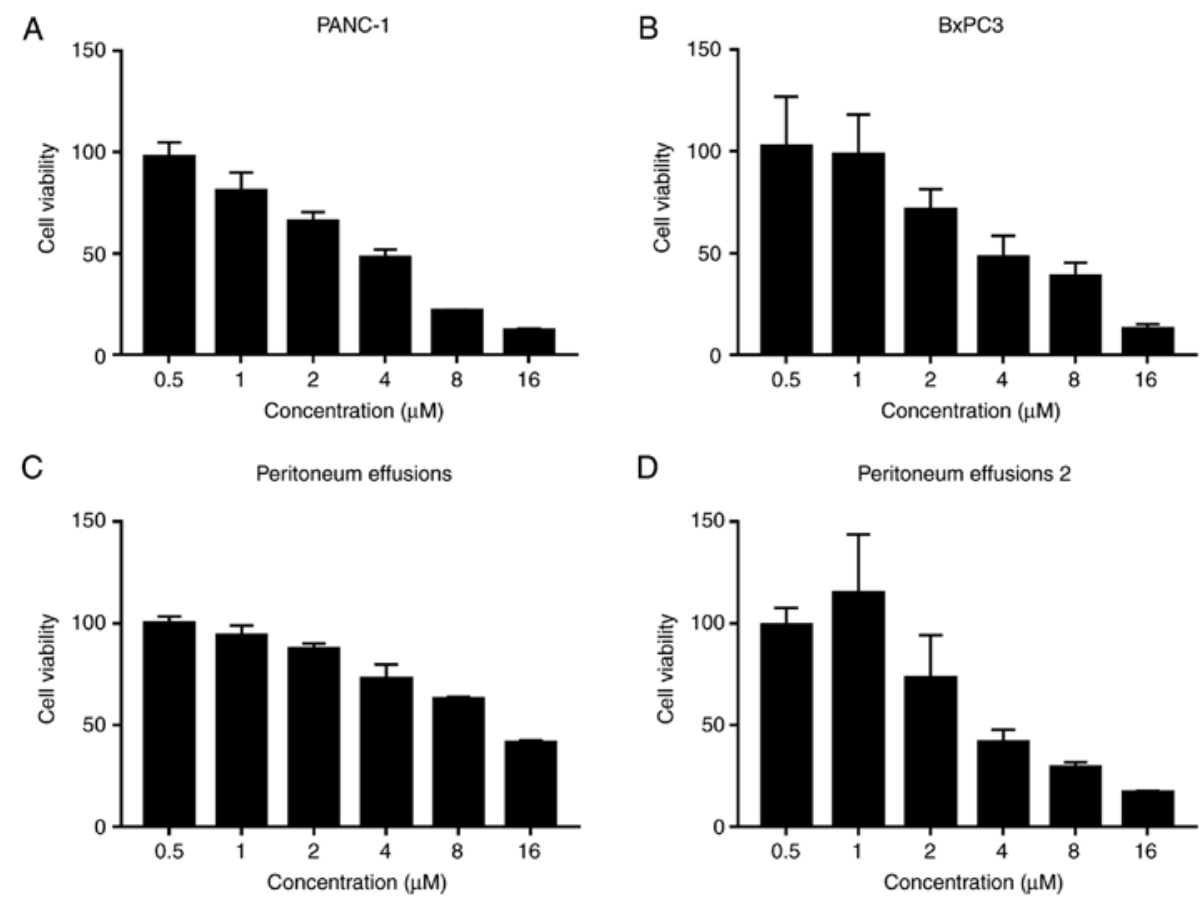

Figure 6. Antitumor effect of $\beta$-elemene in pancreatic cancer. (A) MTT assay revealed that cell viability was suppressed by $\beta$-elemene in PANC-1 cells in a dose-dependent manner. (B) MTT assay revealed that cell viability was suppressed by $\beta$-elemene in BxPC3 cells in a dose-dependent manner. (C and D) MTT assay revealed that cell viability was suppressed by $\beta$-elemene in cells isolated from peritoneum effusion of pancreatic cancer patients in a dose-dependent manner.
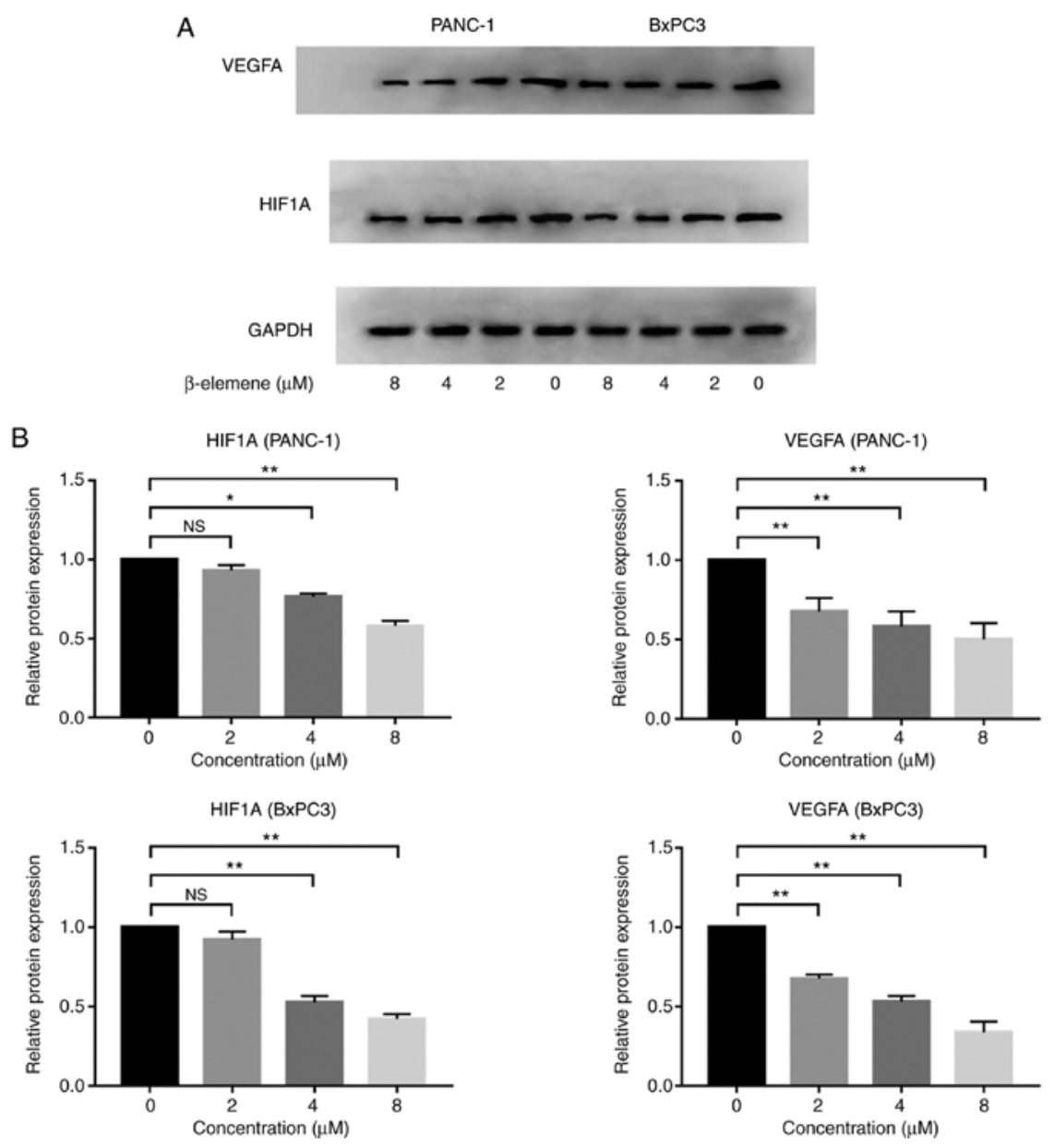

Figure 7. Validation of the HIF1A-VEGFA pathway inhibited by $\beta$-elemene in pancreatic cancer. (A) Western blot analysis in PANC-1 and BxPC3 pancreatic cancer cell lines and (B) corresponding histogram of protein expression levels of HIF1A and VEGFA in PANC-1 and BxPC3 pancreatic cancer cells. Three independent biological replicates were performed and data are presented as the mean \pm standard deviation. ${ }^{*} \mathrm{P}<0.05$ and ${ }^{* * *} \mathrm{P}<0.01$. NS, not significant. HIF1A, hypoxia-inducible factor 1- $\alpha$; VEGFA, vascular endothelial growth factor A. 

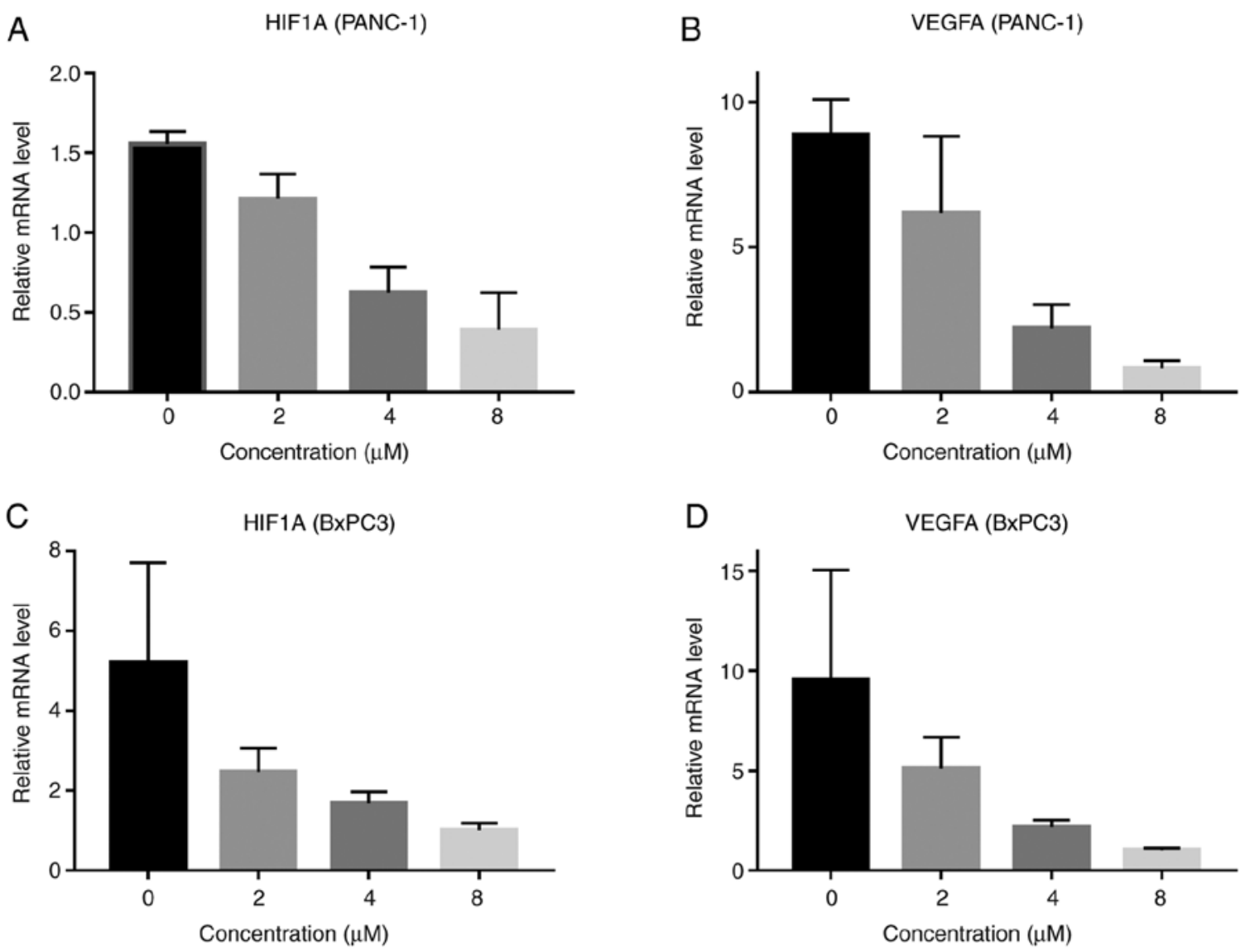

Figure 8. Evaluation of expression of HIF1A and VEGFA at the gene level. RT-qPCR analysis of (A) HIF1A and (B) VEGFA expression in the PANC-1 pancreatic cancer cell line. RT-qPCR analysis of (C) HIF1A and (D) VEGFA expression in the BxPC3 pancreatic cancer cell line. HIF1A, hypoxia-inducible factor 1- $\alpha$; VEGFA, vascular endothelial growth factor A.

effusion in pancreatic cancer, and established a foundation for subsequent studies.

In other studies, $\beta$-elemene was also shown to have a negative impact on HIF1A expression. In one study, the expression level of HIF1A was decreased in A549 cells treated with $\beta$-elemene and radiation compared to the corresponding groups receiving only radiation treatment (22-24). In addition, knockdown of bcl-2-associated transcription factor 1 in hepatocellular carcinoma cell lines significantly reduced the expression of HIF1A, leading to decreased transcription of VEGFA, which in turn suppressed proliferation of hepatocellular carcinoma cells (17). Similarly, rhaponticin exhibits potent anti-metastatic and anti-angiogenic activities due to the inhibition of VEGFA as a consequence of HIF1A suppression (49). Previously, several studies have demonstrated that the HIF1A/VEGFA pathway is related to peritoneum effusion generation (19-21). For instance, peritoneum effusion is enhanced with elevated VEGFA expression and HIF-related hypoxic response (19). Furthermore, lysophosphatidic acid contributes to the generation of peritoneum effusion in ovarian cancer patients by stimulating HIF1A and VEGFA expression through activation of the c-Myc and Sp-1 transcription factors (21). Taken together, our results reveal a possible molecular mechanism of $\beta$-elemene activity against peritoneum effusion, and further highlight the importance of targeting the HIF1A-VEGFA pathway as a therapeutic approach to treat peritoneum effusion in pancreatic cancer patients. However, there exists several limitations in this study. In the first place, the complicated mediation of HIF1A-VEGFA involves multiple pathways regulating the entire process. We only proved the $\beta$-elemene could affect the expression of HIF1A and VEGFA at the protein and mRNA levels. In addition, $\beta$-elemene could induce cytotoxicity via other mechanisms, including apoptosis. All of these diverse aspects of $\beta$-elemene warrant further exploration in future research.

In conclusion, we identified the HIF1A/VEGFA signaling pathway as a promising target relevant to the mechanism of action of $\beta$-elemene in ameliorating malignant peritoneum effusion in pancreatic cancer via a network pharmacology-based strategy. Docking simulation demonstrated the direct binding potential between HIF1A and $\beta$-elemene. Preliminary in vitro studies have been conducted to prove the antitumor effect of $\beta$-elemene and its potential to lower the expression levels of HIF1A and VEGFA, indicating that $\beta$-elemene could mediate the expression of HIF1A and affect its downstream response. Our study provides the theoretical foundation for the application of $\beta$-elemene to treat malignant peritoneum effusion in the clinic. Further research is required for deeper exploration of the antitumor mechanism of $\beta$-elemene moving forward.

\section{Acknowledgements}

We sincerely express our gratitude to Polaris Biology (Shanghai, China) and Dr Zhi Yang for their heartful and prompt assistance in this study. 


\section{Funding}

Not applicable.

\section{Availability of data and materials}

The datasets and materials used and/or analyzed in this study are available from the corresponding author on reasonable request.

\section{Authors' contributions}

JZ and BL contributed equally to this work. Both designed, conducted, and drafted the major parts of this manuscript. YJ, LZ and YZ participated in the network pharmacology analysis, including searching for target genes and constructing individual networks. HZ proposed the idea for this study and facilitated and solved the difficulties encountered in this research. All authors read and approved the final manuscript and agree to be accountable for all aspects of the research in ensuring that the accuracy or integrity of any part of the work are appropriately investigated and resolved.

\section{Ethics approval and consent to participate}

This study was approved by the Internal Review and Ethics Boards of Huadong Hospital Affiliated to Fudan University (Shanghai, China). Fresh samples were collected from two patients upon obtaining written informed consent in October 2018.

\section{Patient consent for publication}

Not applicable.

\section{Competing interests}

The authors declare that they have no competing interests.

\section{References}

1. Bray F, Ferlay J, Soerjomataram I, Siegel RL, Torre LA and Jemal A: Global cancer statistics 2018: GLOBOCAN estimates of incidence and mortality worldwide for 36 cancers in 185 countries. CA Cancer J Clin 68: 394-424, 2018.

2. Kamisawa T, Wood LD, Itoi T and Takaori K: Pancreatic cancer. Lancet 388: 73-85, 2016.

3. Vivaldi C, Caparello C, Musettini G, Pasquini G, Catanese S, Fornaro L, Lencioni M, Falcone A and Vasile E: First-line treatment with FOLFOXIRI for advanced pancreatic cancer in clinical practice: Patients' outcome and analysis of prognostic factors. Int J Cancer 139: 938-945, 2016.

4. Takahara N, Isayama H, Nakai Y, Ishigami H, Satoi S, Mizuno S, Kogure H, Matsubara S, Yamamoto N, Yamaguchi $\mathrm{H}$, et al: Intravenous and intraperitoneal paclitaxel with S-1 for treatment of refractory pancreatic cancer with malignant ascites. Investigational New Drugs 34: 636-642, 2016.

5. Thomassen I, Lemmens VE, Nienhuijs SW, Luyer MD Klaver YL and de Hingh IH: Incidence, prognosis, and possible treatment strategies of peritoneal carcinomatosis of pancreatic origin: A population-based study. Pancreas 42: 72-75, 2013.

6. Xu HB, Zheng LP, Li L, Xu LZ and Fu J: Elemene, one ingredient of a Chinese herb, against malignant tumors: A literature-based meta-analysis. Cancer Invest 31: 156-166, 2013.

7. Chang Z, Gao M,Zhang W, Song L, Jia Y and Qin Y: Beta-elemene treatment is associated with improved outcomes of patients with esophageal squamous cell carcinoma. Surg Oncol 26: 333-337, 2017.
8. Jiang X, Hidru TH, Zhang Z, Bai Y, Kong L and Li X: Evidence of elemene injection combined radiotherapy in lung cancer treatment among patients with brain metastases: A systematic review and meta-analysis. Medicine (Baltimore) 96: e6963, 2017.

9. Feng HB, Wang J, Jiang HR, Mei X, Zhao YY, Chen FR, Qu Y, Sai K, Guo CC, Yang QY, et al: $\beta$-elemene selectively inhibits the proliferation of glioma stem-like cells through the downregulation of Notch1. Stem Cells Transl Med 6: 830-839, 2017.

10. Liu Y, Jiang ZY, Zhou YL, Qiu HH, Wang G, Luo Y, Liu JB, Liu XW, Bu WQ, Song J, et al: $\beta$-elemene regulates endoplasmic reticulum stress to induce the apoptosis of NSCLC cells through PERK/IRE1 $\alpha / A T F 6$ pathway. Biomed Pharmacother 93: 490-497, 2017.

11. Sun W, Huang Y, Yin T, Wang J, Du R, Qiu J, Zhang Y, Wang Y, Chen J and Wang G: Effects of elemene on inhibiting proliferation of vascular smooth muscle cells and promoting reendothelialization at the stent implantation site. Biomater Sci 5: 1144-1155, 2017.

12. Jiang Z, Jacob JA, Loganathachetti DS, Nainangu $P$ and Chen B: $\beta$-elemene: Mechanistic studies on cancer cell interaction and its chemosensitization effect. Front Pharmacol 8: 105, 2017.

13. Li X, Lin Z, Zhang B, Guo L, Liu S, Li H, Zhang J and Ye Q: $\beta$-elemene sensitizes hepatocellular carcinoma cells to oxaliplatin by preventing oxaliplatin-induced degradation of copper transporter 1. Sci Rep 6: 21010, 2016.

14. Wang QT, Zhang ZL, Xiong H, Zhou DS, Li J, Liang J and Wang YF: Evaluation of the efficacy and safety of elemene in treating malignant pleural effusion caused by tumors: A PRISMA guided meta-analysis. Medicine (Baltimore) 97: e12542, 2018.

15. Wang J, Zhang $\mathrm{H}$ and Sun Y: Phase III clinical trial of elemenum emulsion in the management of malignant pleural and peritoneal effusions. Zhonghua Zhong Liu Za Zhi 18: 464-467, 1996 (In Chinese).

16. Hu LP, Zhang XX, Jiang SH, Tao LY, Li Q, Zhu LL, Yang MW, Huo YM, Jiang YS, Tian GA, et al: Targeting purinergic receptor P2Y2 prevents the growth of pancreatic ductal adenocarcinoma by inhibiting cancer cell glycolysis. Clin Cancer Res 25: 1318-1330, 2019.

17. Wen Y, Zhou X, Lu M, He M, Tian Y, Liu L, Wang M, Tan W, Deng Y, Yang X, et al: Bclaf1 promotes angiogenesis by regulating $\mathrm{HIF}-1 \alpha$ transcription in hepatocellular carcinoma. Oncogene 38: 1845-1859, 2019.

18. Li AG, Murphy EC, Culhane AC, Powell E, Wang H, Bronson RT, Von T, Giobbie-Hurder A, Gelman RS, Briggs KJ, et al: BRCA1-IRIS promotes human tumor progression through PTEN blockade and HIF-1 $\alpha$ activation. Proc Natl Acad Sci USA 115: E9600, 2018.

19. Pérez S, Pereda J, Sabater L and Sastre J: Pancreatic ascites hemoglobin contributes to the systemic response in acute pancreatitis. Free Radic Biol Med 81: 145-155, 2015.

20. Pasquet M, Golzio M, Mery E, Rafii A, Benabbou N, Mirshahi P, Hennebelle I, Bourin P, Allal B, Teissie J, et al: Hospicells (ascites-derived stromal cells. promote tumorigenicity and angiogenesis. Int J Cancer 126: 2090-2101, 2010.

21. Song Y, Wu J, Oyesanya RA, Lee Z, Mukherjee A and Fang X: Sp-1 and c-Myc mediate lysophosphatidic acid-induced expression of vascular endothelial growth factor in ovarian cancer cells via a hypoxia-inducible factor-1-independent mechanism. Clin Cancer Res 15: 492-501, 2009.

22. Zou K, Tong E, Xu Y, Deng X and Zou L: Down regulation of mammalian target of rapamycin decreases HIF-1 $\alpha$ and survivin expression in anoxic lung adenocarcinoma A549 cell to elemene and/or irradiation. Tumor Biol 35: 9735-9741, 2014.

23. Tong E, Xu Y, Li G, Zou K and Zou L: The effects of $\beta$-elemene on the expression of mTOR, HIF-1A, survivin in lung adenocarcinoma A549 cell. Afr J Tradit Complement Altern Med 10: $18-23,2013$.

24. Li G, Xie B, Li X, Chen Y, Wang Q, Xu Y, Xu-Welliver M and Zou L: Down-regulation of survivin and hypoxia-inducible factor- $1 \alpha$ by $\beta$-elemene enhances the radiosensitivity of lung adenocarcinoma xenograft. Cancer Biother Radiopharm 27: 56-64, 2012.

25. Liu Z, Guo F, Wang Y, Li C, Zhang X, Li H, Diao L, Gu J, Wang W, Li D, et al: BATMAN-TCM: A bioinformatics analysis tool for molecular mechANism of traditional Chinese medicine. Sci Rep 6: 21146, 2016.

26. Kim J, So S, Lee HJ, Park JC, Kim JJ and Lee H: DigSee: Disease gene search engine with evidence sentences (version cancer). Nucleic Acids Res 41: W510-W517, 2013. 
27. Kim J, Kim JJ and Lee H: An analysis of disease-gene relationship from Medline abstracts by DigSee. Sci Rep 7: 40154-40154, 2017.

28. Szklarczyk D, Morris JH, Cook H, Kuhn M, Wyder S, Simonovic M, Santos A, Doncheva NT, Roth A, Bork P, et al: The STRING database in 2017: Quality-controlled protein-protein association networks, made broadly accessible. Nucleic Acids Res 45: D362-D368, 2017.

29. Huang da W, Sherman BT and Lempicki RA: Systematic and integrative analysis of large gene lists using DAVID bioinformatics resources. Nature Protoc 4: 44-57, 2008.

30. Huang da W, Sherman BT and Lempicki RA: Bioinformatics enrichment tools: Paths toward the comprehensive functional analysis of large gene lists. Nucleic Acids Res 37: 1-13, 2009.

31. Shannon P, Markiel A, Ozier O, Baliga NS, Wang JT, Ramage D, Amin N, Schwikowski B and Ideker T: Cytoscape: A software environment for integrated models of biomolecular interaction networks. Genome Res 13: 2498-2504, 2003.

32. Goodsell DS and Olson AJ: Automated docking of substrates to proteins by simulated annealing. Proteins 8 : 195-202, 1990.

33. Schrödinger LLC: The PyMOL molecular graphics system, version 1.8, 2015.

34. Tomczak K, Czerwińska P and Wiznerowicz M: The Cancer Genome Atlas (TCGA): An immeasurable source of knowledge. Contemp Oncol (Pozn) 19: A68-A77, 2015.

35. Gao J, Aksoy BA, Dogrusoz U, Dresdner G, Gross B, Sumer SO, Sun Y, Jacobsen A, Sinha R, Larsson E, et al: Integrative analysis of complex cancer genomics and clinical profiles using the cBioPortal. Sci Signal 6: pl1, 2013.

36. Cerami E, Gao J, Dogrusoz U, Gross BE, Sumer SO, Aksoy BA, Jacobsen A, Byrne CJ, Heuer ML, Larsson E, et al: The cBio cancer genomics portal: An open platform for exploring multidimensional cancer genomics data. Cancer Discov 2: 401-404, 2012.

37. Lánczky A, Nagy Á, Bottai G, Munkácsy G, Szabó A, Santarpia L and Győrffy B: miRpower: A web-tool to validate survival-associated miRNAs utilizing expression data from 2178 breast cancer patients. Breast Cancer Res Treat 160 439-446, 2016.

38. An YW, Hu G, Yin GP, Zhu JJ, Zhang QW, Wang ZM, Peng J and Fan B: Quantitative analysis and discrimination of steamed and non-steamed rhizomes of Curcuma wenyujin by GC-MS and HPLC. J Chromatogr Sci 52: 961-970, 2014.

39. Tang CY, Zhu LX, Yu JD, Chen Z, Gu MC, Mu CF, Liu Q and Xiong Y: Effect of $\beta$-elemene on the kinetics of intracellular transport of d-luciferin potassium salt (ABC substrate) in doxorubicin-resistant breast cancer cells and the associated molecular mechanism. Eur J Pharm Sci 120: 20-29, 2018.
40. Li X, Wang G, Zhao J, Ding H, Cunningham C, Chen F, Flynn DC, Reed $\mathrm{E}$ and Li QQ: Antiproliferative effect of beta-elemene in chemoresistant ovarian carcinoma cells is mediated through arrest of the cell cycle at the G2-M phase. Cell Mol Life Sci 62: 894-904, 2005

41. Liu JS, Che XM, Chang S, Qiu GL, He SC, Fan L, Zhao W, Zhang ZL and Wang SF: $\beta$-elemene enhances the radiosensitivity of gastric cancer cells by inhibiting Pak1 activation. World J Gastroenterol 21: 9945-9956, 2015.

42. Dai ZJ, Tang W, Lu WF, Gao J, Kang HF, Ma XB, Min WL, Wang XJ and Wu WY: Antiproliferative and apoptotic effects of $\beta$-elemene on human hepatoma HepG2 cells. Cancer Cell Int 13: 27, 2013.

43. Cheng H, Ge X, Zhuo S, Gao Y, Zhu B, Zhang J, Shang W, $\mathrm{Xu} D, \mathrm{Ge} W$ and Shi L: $\beta$-elemene synergizes with gefitinib to inhibit stem-like phenotypes and progression of lung cancer via down-regulating EZH2. Front Pharmacol 9: 1413, 2018.

44. Hopkins AL: Network pharmacology: The next paradigm in drug discovery. Nat Chem Biol 4: 682-690, 2008.

45. Zheng J, Wu M, Wang H, Li S, Wang X, Li Y, Wang D and Li S: Network pharmacology to unveil the biological basis of health-strengthening herbal medicine in cancer treatment. Cancers (Basel) 10: E461, 2018.

46. Wang N, Yang B, Zhang X, Wang S, Zheng Y, Li X, Liu S, Pan H, $\mathrm{Li} Y$, Huang Z, et al: Network pharmacology-based validation of caveolin-1 as a key mediator of Ai Du Qing inhibition of drug resistance in breast cancer. Front Pharmacol 9: 1106, 2018.

47. Xing X, Chen S, Li L, Cao Y, Chen L, Wang X and Zhu Z: The active components of Fuzheng Huayu formula and their potential mechanism of action in inhibiting the hepatic stellate cells viability-a network pharmacology and transcriptomics approach Front Pharmacol 9: 525, 2018.

48. Wang Y, Guo G, Yang BR, Xin QQ, Liao QW, Lee SM, $\mathrm{Hu} \mathrm{YJ}$, Chen KJ and Cong WH: Synergistic effects of Chuanxiong-Chishao herb-pair on promoting angiogenesis at network pharmacological and pharmacodynamic levels. Chin J Integr Med 23: 654-662, 2017.

49. Kim A and Ma JY: Rhaponticin decreases the metastatic and angiogenic abilities of cancer cells via suppression of the HIF-1a pathway. Int J Oncol 53: 1160-1170, 2018.

This work is licensed under a Creative Commons Attribution-NonCommercial-NoDerivatives 4.0 International (CC BY-NC-ND 4.0) License. 